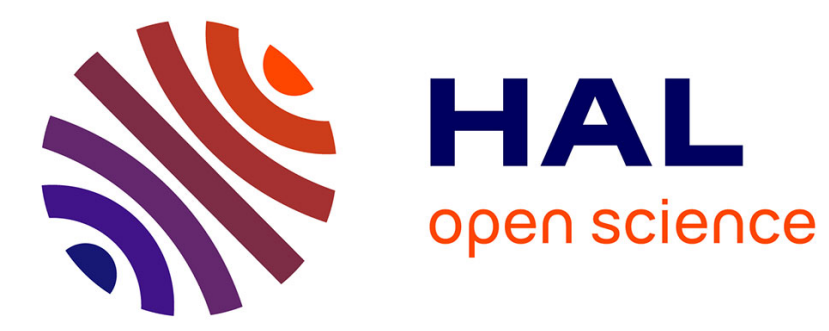

\title{
Robust stability of impulsive systems: A functional-based approach
}

\author{
Corentin Briat, Alexandre Seuret
}

\section{To cite this version:}

Corentin Briat, Alexandre Seuret. Robust stability of impulsive systems: A functional-based approach. ADHS 2012 - 4th IFAC conference on Analysis and Design of Hybrid Systems, Jun 2012, Eindhoven, Netherlands. pp.6, 10.3182/20120606-3-NL-3011.00064 . hal-00679207

\section{HAL Id: hal-00679207 https://hal.science/hal-00679207}

Submitted on 15 Mar 2012

HAL is a multi-disciplinary open access archive for the deposit and dissemination of scientific research documents, whether they are published or not. The documents may come from teaching and research institutions in France or abroad, or from public or private research centers.
L'archive ouverte pluridisciplinaire HAL, est destinée au dépôt et à la diffusion de documents scientifiques de niveau recherche, publiés ou non, émanant des établissements d'enseignement et de recherche français ou étrangers, des laboratoires publics ou privés. 


\title{
Robust stability of impulsive systems: A functional-based approach
}

\author{
C. Briat* A. Seuret ${ }^{* *}$ \\ * Department of Biosystems Science and Engineering (D-BSSE), Swiss \\ Federal Institute of Technology-Zurich (ETHZ), Mattenstrasse 26, \\ 4058 Basel, Switzerland, corentin@briat.info, \\ briatc@bsse.ethz.ch \\ ** Department of Automatic Control, Gipsa-lab, 961 rue de la Houille \\ Blanche, $\mathrm{BP}$ 46, 38402 Grenoble Cedex, France, \\ alexandre.seuret@gipsa-lab.grenoble-inp.fr
}

\begin{abstract}
An improved functional-based approach for the stability analysis of linear uncertain impulsive systems relying on Lyapunov looped-functionals is provided. Looped functionals are peculiar functionals that allow to encode discrete-time stability criteria into continuous-time conditions and to consider non-monotonic Lyapunov functions along the trajectories of the impulsive system. Unlike usual discrete-time stability conditions, the obtained ones are convex in the system matrices, an important feature for extending the results to uncertain systems. It is emphasized in the examples that the proposed approach can be applied to a class of systems for which existing approaches are inconclusive, notably systems having unstable continuous and discrete dynamics.
\end{abstract}

Keywords: Impulsive systems, Lyapunov looped functionals, Uncertain systems, LMIs

\section{INTRODUCTION}

Impulsive systems [Bainov and Simeonov, 1989, Dullerud and Lall, 1999, Yang, 2001, Cai et al., 2008, Hespanha et al., 2008, Michel et al., 2008, Goebel et al., 2009] are an important class of hybrid systems in which the system trajectory admits discontinuities at certain timeinstants. They occur in several fields like epidemiology [Stone et al., 2000, Briat and Verriest, 2009], sampled-data and networked control systems [Naghshtabrizi et al., 2008, Sun et al., 1991, Sivashankar and Khargonekar, 1994], etc. Among the wide class of impulsive dynamical systems, we may differentiate systems whose impulse-times depend on the system state and those for which the impulse-times are external to system and only time-dependent. The latter class may be represented in the following form

$$
\begin{aligned}
& \dot{x}(t)=A x(t), t \in \mathbb{R}_{+} \backslash \mathbb{I}, \\
& x\left(t^{+}\right)=J x(t), t \in \mathbb{I},
\end{aligned}
$$

where $x \in \mathbb{R}^{n}$ is the state of the system and the matrices $A$ and $J$ are matrices of appropriate dimensions. These matrices are assumed to be perfectly known in Section 3 , then uncertain in Section 4. The sequence of impulse instants $\left\{t_{k}\right\}_{k_{1} \mathbb{N}}$ is assumed to be increasing and to have no accumulation point. The state-trajectory is considered as left-continuous and the right limit at a point of discontinuity $t_{k}$ is denoted by $x\left(t_{k}^{+}\right)=\lim _{s \downarrow t_{k}} x(s)$. Depending on the structure of the matrices $A$ and $J$, the system may exhibit very different behaviors. In particular, notions of minimal and maximal dwell-time can be defined for impulsive systems [Hespanha et al., 2008], similarly as for switched systems [Liberzon, 2003, Geromel and Colaneri, 2006]. In the case of impulsive systems, these notions refer to system properties such that too large or too short inter- impulse intervals destabilize the system. In the case of periodic impulses with period $T>0$, the problem essentially reduces to the study of the Schurness of the matrix $J e^{A T}$, which turns out to be a very simple problem. However, this formulation suffers from several critical drawbacks since it cannot be easily extended to the case of aperiodic impulses and uncertain systems.

The approach discussed in this paper aims at overcoming these important drawbacks. The underlying idea has been triggered by the recent work [Naghshtabrizi et al., 2008] where an impulsive model is used for stability analysis of aperiodic sampled-data systems [Sun et al., 1991, Sivashankar and Khargonekar, 1994] and studied using Lyapunov functionals. This idea has been later improved by one of the author in [Seuret, 2012] where an implicit but equivalent correspondence between discrete- and continuous-time domains is obtained. It is shown there that discrete-time stability is equivalent to a certain kind of continuous-time stability, provided that the latter is proved using peculiar functionals referred to as loopedfunctionals. Based on this approach, some results have been obtained in [Briat and Seuret, 2012]. The interest for considering discrete-time criteria lies in the fact that a discrete-time condition is a much weaker condition than a continuous-time one which demands the monotonic decrease of a positive function. A discrete-time condition requires the decrease of a sequence of points only.

On the top of this, the proposed functional-based approach leads to LMI-conditions which are affine in the interimpulse period and convex in the system matrices $A$ and $J$, hence easily extendable to the cases of aperiodic impulses 
and uncertain systems. Notably, stability under ranged dwell-time, i.e. $t_{k+1}-t_{k} \in\left[T_{\min }, T_{\max }\right]$, is considered.

Outline: Section 2 introduces some preliminaries. Sections 3 and 4 are devoted to nominal and robust stability analysis. Illustrative examples are included in the related sections.

Notations: for symmetric matrices $A, B, A-B \prec(\preceq) 0$ means that $A-B$ negative (semi)definite. The sets of symmetric and positive definite matrices of dimension $n$ are denoted by $\mathbb{S}^{n}$ and $\mathbb{S}_{+}^{n}$ respectively. The spectral radius of a square matrix $M$ is denoted by $\rho(M)$. Given a square matrix $M$, we define $\operatorname{He}[M]=M+M^{T}$.

\section{PRELIMINARIES}

\subsection{A general stability result}

The proposed approach relies on the characterization of system (1) using a lifting approach [Yamamoto, 1990] since it is based on a discrete-time criterion expressed in continuous-time. It is hence necessary to consider the state-space $\mathbb{K}$ consisting of the set of continuous-functions with varying support. The elements are then of the form

$$
\chi_{k}:\left(0, T_{k}\right] \rightarrow \mathbb{R}^{n},
$$

where $T_{k}=t_{k+1}-t_{k}<\infty, k \in \mathbb{N}$. The new state-space is hence infinite-dimensional and verifies

$$
\begin{aligned}
& \chi_{k}(\tau):=x\left(t_{k}+\tau\right), \tau>0 \\
& \chi_{k}(\tau)=e^{A \tau} \chi_{k}\left(0^{+}\right), \\
& \chi_{k}\left(0^{+}\right)=J \chi_{k-1}\left(T_{k-1}\right)=J x\left(t_{k}\right) .
\end{aligned}
$$

The idea for proving stability is to look for a positive definite quadratic form $V(x)$ such that the sequence $\left\{V\left(x\left(t_{k}\right)\right)\right\}_{k \in \mathbb{N}}$ is monotonically decreasing ${ }^{1}$. This is formalized below through a functional existence result:

Theorem 1. Let $\varepsilon<T_{\min } \leq T_{\max }<+\infty$ be three positive scalars and $V: \mathbb{R}^{n} \rightarrow \mathbb{R}^{+}$be a quadratic function verifying

$$
\forall x \in \mathbb{R}^{n}, \quad \mu_{1}\|x\|_{2}^{2} \leq V(x) \leq \mu_{2}\|x\|_{2}^{2},
$$

for some scalars $0<\mu_{1}<\mu_{2}$. Then, the following statements are equivalent.

(i) The sequence $\left\{V\left(x\left(t_{k}\right)\right)\right\}_{k \in \mathbb{N}}$ is decreasing; that is $V(x)$ is a discrete-time Lyapunov function for the system $x\left(t_{k+1}\right)=e^{A T_{k}} J x\left(t_{k}\right), T_{k} \in\left[T_{\min }, T_{\max }\right]$;

(ii) There exists a differentiable functional $\mathcal{V}:\left[0, T_{\text {max }}\right] \times$ $\mathbb{K} \rightarrow \mathbb{R}$ satisfying

$$
\mathcal{V}(\theta, z)=\mathcal{V}\left(0^{+}, z\right)
$$

for all $z \in \mathbb{K}$ and for all $\theta \in\left[T_{\min }, T_{\max }\right]$ and a function $V_{0}: \mathbb{R}^{n} \rightarrow \mathbb{R}$ such that

$$
\dot{\mathcal{W}}_{k}\left(\tau, \chi_{k}\right)=\Lambda_{k}+\frac{\mathrm{d}}{\mathrm{d} \tau}\left[T_{k} \bar{V}\left(\chi_{k}(\tau)\right)+\mathcal{V}\left(\tau, \chi_{k}\right)\right]<0,
$$

holds for all $\tau \in\left[0, T_{k}\right], T_{k} \in\left[T_{\min }, T_{\max }\right], k \in \mathbb{N}$ and where

$$
\begin{aligned}
\bar{V}\left(\chi_{k}(\tau)\right) & =V\left(\chi_{k}(\tau)\right)+V_{0}\left(\chi_{k}(\tau)\right) \\
\Lambda_{k} & =\bar{V}\left(\chi_{k}\left(0^{+}\right)\right)-V\left(\chi_{k-1}\left(T_{k-1}\right)\right)-V_{0}\left(\chi_{k}\left(T_{k}\right)\right) .
\end{aligned}
$$

Moreover, if one of the previous statement is satisfied, the impulsive system (1) is asymptotically stable.

\footnotetext{
1 We may also look at the sequence $\left\{V\left(x\left(t_{k}^{+}\right)\right)\right\}_{k \in \mathbb{N}}$ instead. The choice is purely arbitrary.
}

Proof : $\quad(\mathbf{i i}) \Rightarrow(\mathbf{i})$ : Let $k \in \mathbb{N}, \tau \in\left[0, T_{k}\right]$ and $T_{k} \in$ $\left[T_{\min }, T_{\max }\right]$. Assume that $(i i)$ is satisfied. Integrating $\dot{\mathcal{W}}$ yields

$$
\begin{aligned}
\int_{0}^{T_{k}} \dot{\mathcal{W}}_{k}\left(\tau, \chi_{k}\right) \mathrm{d} \tau= & T_{k}\left(\Lambda_{k}+\bar{V}\left(\chi_{k}\left(T_{k}\right)\right)-\bar{V}\left(\chi_{k}\left(0^{+}\right)\right)\right) \\
& +\mathcal{V}\left(T_{k}, \chi_{k}\right)-\mathcal{V}\left(0^{+}, \chi_{k}\right) .
\end{aligned}
$$

The last row vanishes according to (2) and noting then that $\chi_{k}\left(0^{+}\right)=x\left(t_{k}^{+}\right), \chi_{k-1}\left(T_{k-1}\right)=x\left(t_{k}\right), \chi_{k}\left(T_{k}\right)=$ $x\left(t_{k+1}\right)$, we get

$$
\begin{aligned}
\int_{0}^{T_{k}} \dot{\mathcal{W}}_{k}\left(\tau, \chi_{k}\right) \mathrm{d} \tau= & T_{k}\left[V\left(x\left(t_{k}^{+}\right)\right)-V\left(x\left(t_{k}\right)\right)\right. \\
& \left.+V\left(x\left(t_{k+1}\right)\right)-V\left(x\left(t_{k}^{+}\right)\right)\right] \\
& +T_{k}\left[V_{0}\left(x\left(t_{k+1}\right)\right)-V_{0}\left(x\left(t_{k}^{+}\right)\right)\right] \\
& -T_{k}\left[V_{0}\left(x\left(t_{k+1}\right)\right)-V_{0}\left(x\left(t_{k}^{+}\right)\right)\right] \\
= & T_{k}\left[V\left(x\left(t_{k+1}\right)\right)-V\left(x\left(t_{k}\right)\right)\right] .
\end{aligned}
$$

Then, the sequence $\left\{V\left(x\left(t_{k}\right)\right)\right\}_{k \in \mathbb{N}}$ is decreasing over $k$ since $\dot{\mathcal{W}}_{k}$ is negative over $\left[0, T_{k}\right]$.

(i) $\Rightarrow$ (ii): Assume that $(i)$ is satisfied. Similarly as in [Seuret, 2012], introduce the functional $\mathcal{V}\left(\tau, \chi_{k}(\tau)\right)=$ $-T_{k} V\left(\chi_{k}(\tau)\right)+\tau\left(V\left(x\left(t_{k+1}\right)\right)-V\left(x\left(t_{k}\right)\right)\right)$ and choosing $V_{0}\left(\chi_{k}(\tau)\right)=0$. Simple computations show that conditions (2) holds and that $\dot{\mathcal{W}}_{k}\left(\tau, \chi_{k}\right)=V\left(x\left(t_{k+1}\right)\right)-V\left(x\left(t_{k}\right)\right)<0$. Equivalence is proved.

It remains to prove that $V(x(t))$ (or equivalently $V\left(\chi_{k}(\tau)\right)$ ) is bounded over $\left(t_{k}, t_{k+1}\right]$. Noting that $x\left(t_{k}+\tau\right)=$ $e^{A \tau} J x\left(t_{k}\right), \tau>0$, then boundedness immediately follows. The proof is complete.

The functional $\mathcal{W}$ is a new type of functionals referred to as looped-functional, in accordance with the fact that the boundary condition loops the functional to the same value on the boundary of the support of the functional state, i.e. $\left[0, T_{k}\right]$. There is a fundamental difference with existing methods based on functionals relying on Lyapunov's theorem which demands the positivity of the functional. In the proposed approach, the positivity requirement is relaxed and substituted by the boundary condition. The only positivity requirement holds on the term $V$, the only term on which the Lyapunov's theorem must be applied. The relaxation of the sign requirement may seem to violate Lyapunov's theorem, but it should be kept in mind here that the proposed stability result is a discrete-time stability result expressed in continuous-time, a rather different way of thinking about the problem with its own conditions, different from those of the standard continuous-time ones.

Considering a discrete-time stability condition is much weaker than a continuous-time one since the continuoustime Lyapunov function is not necessarily monotonically decreasing along the trajectories of the system anymore. This feature is extremely important in the current framework in order to cope with expansive jumps and unstable continuous-time dynamics. Using such a discrete-time approach, only the decrease of the function evaluated at impulse-instants is important.

The second point of the approach concerns the fact that the continuous-time and discrete-time parts of the systems are merged together into the single condition $\dot{\mathcal{W}}<0$, instead of two disjoint ones as in most of the works on 
impulsive systems, see e.g. [Cai et al., 2008, Hespanha et al., 2008, Goebel et al., 2009]. It is hence expected to embed more information on the behavior of the impulsive systems by capturing possible close interactions between the discrete-time and continuous-time parts, e.g. those carried by the eigenvectors. Note that such interactions seem difficult to handle using distinct continuous and discrete conditions, due to the covering effect of the Lyapunov function which masks the internal behavior of the system. It is indeed shown in the examples that the proposed approach is able to characterize stability for a larger class of systems than existing methods [Cai et al., 2008, Hespanha et al., 2008], namely systems for which neither $A$ nor $J$ is stable. For such systems, the admissible dwell-times may be both bounded from above and below. Definition 1 in [Cai et al., 2008] both imposes a continuoustime and discrete-time decrease in the Lyapunov function level, hence assuming the that dynamics are stable, at least at certain time instants. In the proposed approach, it may be assumed that some dynamics are never stable. In [Hespanha et al., 2008], the conditions for dwell-time and reverse dwell-time explicitly assume that at least one of the matrices is stable. In the current approach, this condition is relaxed.

\section{NOMINAL STABILITY ANALYSIS OF LINEAR IMPULSIVE SYSTEMS}

This section provides some nominal stability results for both periodic and aperiodic impulses. In the following, we will make extensive uses of the matrix expression:

$$
\mathcal{I}(P, A, J, \theta):=J^{T} e^{A^{T} \theta} P e^{A \theta} J-P
$$

where $\mathcal{I}$ stands for 'impulsive'.

\subsection{Stability analysis of linear impulsive systems - Periodic impulses case}

An LMI sufficient condition for asymptotic stability of the impulsive system (1) is provided below using the framework defined in Theorem 1:

Theorem 2. The impulsive system (1) with $t_{k+1}-t_{k}=T$, $k \in \mathbb{N}$ is asymptotically stable if there exist matrices $P, Z \in \mathbb{S}_{+}^{n}, P_{0}, S, Q \in \mathbb{S}^{n}, U \in \mathbb{S}^{2 n}, R \in \mathbb{R}^{n \times 2 n}$ and $N \in \mathbb{R}^{n \times 3 n}$ such that the LMIs

$$
\begin{aligned}
\Psi(A, J, T):=F_{0}+T\left(F_{2}+F_{3}\right) & \prec 0, \\
\Phi(A, J, T):=\left[\begin{array}{cc}
F_{0}+T\left(F_{1}-F_{3}\right) & T N^{T} \\
\star & -T Z
\end{array}\right] & \prec 0,
\end{aligned}
$$

hold with

$$
\begin{array}{rlrl}
M_{x} & =\left[\begin{array}{lll}
I & 0 & 0
\end{array}\right], \quad M_{-} & =\left[\begin{array}{lll}
0 & I & 0
\end{array}\right], \\
M_{+} & =\left[\begin{array}{lll}
0 & 0 & I
\end{array}\right], \quad G=\left[\begin{array}{lll}
M_{-}^{T} & M_{+}^{T}
\end{array}\right]^{T}, \\
M_{\zeta} & =\left[\begin{array}{lll}
I & -J & 0
\end{array}\right], \quad M_{\varsigma}=\left[\begin{array}{lll}
I & 0 & -I
\end{array}\right],
\end{array}
$$

and where $F_{3}=G^{T} U G, F_{1}=\operatorname{He}\left[M_{x}^{T} A^{T} S M_{\varsigma}\right]$ and

$$
\begin{aligned}
F_{0}= & T \operatorname{He}\left\{M_{x}^{T}\left(P+P_{0}\right) A M_{x}\right\}-M_{\zeta}^{T} Q M_{\zeta} \\
& +\operatorname{He}\left[-N^{T} M_{\zeta}-M_{\zeta}^{T} R G\right]+M_{\varsigma} S M_{\varsigma} \\
& -M_{+}^{T} P_{0} M_{+}+M_{-}^{T}\left(J^{T}\left(P+P_{0}\right) J-P\right) M_{-}, \\
F_{2}= & \operatorname{He}\left[M_{x}^{T} A^{T} Q M_{\zeta}+M_{x}^{T} A^{T} R G\right] \\
& +M_{x}^{T} A^{T} Z A M_{x} .
\end{aligned}
$$

Moreover, the quadratic function $V(x)=x^{T} P x$ is a discrete-time Lyapunov function for system (1), that is the LMI $\mathcal{I}(P, A, J, T) \prec 0$ holds.
Proof : Choosing $V(x)=x^{T} P x, V_{0}(x)=x^{T} P_{0} x$ and

$$
\begin{aligned}
\mathcal{V}\left(\tau, \chi_{k}\right) & =(T-\tau) \zeta_{k}(\tau)^{T}\left[Q \zeta_{k}(\tau)+2 R\left(\begin{array}{c}
\chi_{k-1}(T) \\
\chi_{k}(T)
\end{array}\right)\right] \\
& +\tau \varsigma_{k}(\tau)^{T} S \varsigma_{k}(\tau)+(T-\tau) \int_{0}^{\tau} \dot{\chi}_{k}(s)^{T} Z \dot{\chi}_{k}(s) d s \\
& +\tau(T-\tau)\left[\begin{array}{c}
\chi_{k-1}(T) \\
\chi_{k}(T)
\end{array}\right]^{T} U\left[\begin{array}{c}
\chi_{k-1}(T) \\
\chi_{k}(T)
\end{array}\right],
\end{aligned}
$$

where $\zeta_{k}(\tau)=\chi_{k}(\tau)-\chi_{k}(0)=\chi_{k}(\tau)-J \chi_{k-1}(T), \varsigma_{k}(\tau)=$ $\chi_{k}(\tau)-\chi_{k}(T), P, Z \in \mathbb{S}_{+}^{n} P_{0}, Q, S \in \mathbb{S}^{n}, R \in \mathbb{R}^{n \times 2 n}$ and $U \in \mathbb{S}^{2 n}$ Differentiation yields

$$
\begin{aligned}
\dot{\mathcal{W}}_{k}:= & T_{k} \dot{\bar{V}}+\dot{\mathcal{V}}+\Lambda_{k} \\
\leq & \xi_{k}(\tau)^{T}\left[F_{0}+\tau F_{1}^{\prime}+(T-\tau) F_{2}\right. \\
& \left.+(T-2 \tau) F_{3}\right] \xi_{k}(\tau),
\end{aligned}
$$

where $F_{1}^{\prime}=F_{1}+N^{T} Z^{-1} N, \xi_{k}(t)=\operatorname{col}\left(\chi_{k}(\tau), \chi_{k-1}(T)\right)$ and

$$
\begin{aligned}
\Lambda_{k}= & \chi_{k-1}(T)^{T}\left(J^{T}\left(P+P_{0}\right) J-P\right) \chi_{k-1}(T) \\
& -\chi_{k}(T)^{T} P_{0} \chi_{k}(T) .
\end{aligned}
$$

To obtain the bound on $\dot{\mathcal{W}}_{k}$, the affine Jensen's bound [Seuret, 2009, Briat, 2011] have been used on the integral term

$$
\begin{aligned}
-\int_{0}^{\tau} \dot{\chi}_{k}(s)^{T} Z \dot{\chi}_{k}(s)(s) d s \leq & \xi_{k}(\tau)^{T}\left(2 N^{T} M_{\zeta}\right. \\
& \left.+\tau N^{T} Z^{-1} N\right) \xi_{k}(\tau) .
\end{aligned}
$$

Since the resulting matrix inequality is affine in $\tau$, to check its feasibility over $[0, T]$, it is necessary and sufficient to check it at the vertices of the set, that is for $\tau \in\{0, T\}$. Finally, a Schur complement on the quadratic term $T N^{T} Z^{-1} N$ yields the result.

It is important to stress that while Theorem 1 provides a necessary and sufficient condition, the above result provides a sufficient one only. The necessity is indeed destroyed when choosing the specific functional (5).

The main improvement of the proposed approach lies in the introduction of information at $\tau=T$ in the Lyapunov looped functional through the terms involving $\chi_{k}(T)$. The use of this additional information is expected to reduce the conservatism of the approach.

\subsection{Stability over arbitrary intervals - Ranged dwell-time}

A sufficient condition for ranged dwell-time is given in terms of the convex robust feasibility problem

$$
\mathcal{I}(P, A, J, \theta) \prec 0,
$$

which must be satisfied for some $P=P^{T} \succ 0$ and all $\theta \in\left[T_{\min }, T_{\max }\right]$. The following theorem provides a sufficient condition for (7), devoid of exponential terms:

Theorem 3. The impulsive system (1) with $T_{k} \in\left[T_{\text {min }}\right.$, $\left.T_{\max }\right], 0<\varepsilon<T_{\min } \leq T_{\max }<\infty, \varepsilon>0$, is asymptotically stable if there exist matrices $P, Z \in \mathbb{S}_{+}^{n}$, $P_{0}, S, Q \in \mathbb{S}^{n}, U \in \mathbb{S}^{2 n}, R \in \mathbb{R}^{n \times 2 n}$ and $N \in \mathbb{R}^{n \times 3 n}$ such that $\Psi(A, J, T) \prec 0$ and $\Phi(A, J, T) \prec 0$ hold for all $T \in\left\{T_{\min }, T_{\max }\right\}$.

In such a case, the following inequality

$$
\mathcal{I}(P, A, J, \theta) \prec 0
$$

holds for all $\theta \in\left[T_{\min }, T_{\max }\right]$ and with the same matrix $P$. 
Proof: Since the LMIs of Theorem 2 are convex in $T$, a convexity argument yields the result.

It seems important to point out that this result is able to prove stability for a quite large class of systems. Indeed, it may applied to systems for which neither $A$ and $J$ are stable. This is to put in contrast for instance with [Hespanha et al., 2008, Theorem 1], transferred to a linear setting, for which it is necessary that one of the matrices be stable.

\subsection{Examples}

Example 1. Let us consider system (1) with matrices

$$
A=\left[\begin{array}{cc}
1 & 3 \\
-1 & 2
\end{array}\right], \quad J=0.5 I_{2}
$$

Note that $A$ has 2 unstable eigenvalues at $1.5 \pm 1.6583 j$, hence we need a stabilizing matrix $J$, i.e. $\rho(J)<1$. Moreover, it is easy to see that if the pulse period is too large, stability is not achievable. Using the spectral radius condition $\rho\left(e^{A T} J\right)<1$, the maximal allowable constant dwell time $T_{\max }^{e i g}=0.4620$ is found.

The results are provided in Table 1 where we can see that the proposed approach improves the results of [Briat and Seuret, 2012] both in the periodic and aperiodic cases.

Example 2. Now consider system (1) with matrices

$$
A=\left[\begin{array}{cc}
-1 & 0 \\
1 & -2
\end{array}\right], \quad J=\left[\begin{array}{ll}
2 & 1 \\
1 & 3
\end{array}\right] \text {. }
$$

In this case, the matrix $A$ is Hurwitz while $J$ is anti-Schur. Hence, if $T$ is to small, stability is lost. It may be seen in Table 1 that the proposed approach yields better results than the approach in [Briat and Seuret, 2012].

Example 3. Consider now system (1) with matrices

$$
A=\left[\begin{array}{cc}
-1 & 0.1 \\
0 & 1.2
\end{array}\right], \quad J=\left[\begin{array}{cc}
1.2 & 0 \\
0 & 0.5
\end{array}\right] \text {. }
$$

In such a case, the continuous-time dynamics of the first state is stable while the second is unstable. On the other hand, the matrix $J$ has a stable eigenvalue for the second state and an unstable eigenvalue for the first one. Therefore, if the period $T$ is too small, a destabilizing pulse will occur too often. Conversely, if the period $T$ is too large, the stabilizing pulse will not be able to stabilize the unstable continuous-time dynamics. It is hence expected to have an admissible period $T$ inside an interval excluding both 0 and $\infty$.

In the periodic case, an eigenvalue analysis yields the bounds 0.1824 and 0.5776 . The results obtained in the periodic and aperiodic cases are summarized in Table 1 where we can see that the proposed functional yields less conservative results.

To emphasize that this system cannot be successfully analyzed using existing methods, let us choose $T=0.3$ and we find $P=\operatorname{diag}(2.3622,1.4752)$ along with

$$
A^{T} P+P A \preceq-c P, \quad J^{T} P J-P \preceq e^{-d} P,
$$

where $c=-2.4036$ and $d=-0.3646$. Since both $c$ and $d$ are negative, the method of [Hespanha et al., 2008] is inconclusive. This fact also applies to the conditions stated in Definition 1 of [Cai et al., 2008]. This is an intrinsic drawback of formulations based on disjoint continuous and discrete conditions. In this case, the Lyapunov function covers some properties of the system and masks possible interactions between the continuous and discrete-time parts. In the current example, the interactions are carried by the eigenvectors, an information irremediably lost when Lyapunov functions are used.

Example 4. Let us consider the example of the sampleddata control system

$$
\begin{aligned}
& \dot{x}(t)=\tilde{A} x(t)+B u(t), \\
& u(t)=K x\left(t_{k}\right), t \in\left[t_{k}, t_{k+1}\right),
\end{aligned}
$$

where $t_{k+1}-t_{k}=T, k \in \mathbb{N}$. Reformulating this system as an impulsive system, we obtain

$$
\dot{z}(t)=\left[\begin{array}{cc}
\tilde{A} & B \\
0 & 0
\end{array}\right] z(t) \quad z\left(t_{k}^{+}\right)=\left[\begin{array}{cc}
I_{n} & 0 \\
K & 0
\end{array}\right] z\left(t_{k}\right)
$$

where $z(t)=\operatorname{col}(x(t), u(t))$. Let us consider for instance a sampled-data system with matrices [Naghshtabrizi et al., 2008, Seuret, 2012, Briat and Seuret, 2011]

$$
\tilde{A}=\left[\begin{array}{cc}
0 & 1 \\
0 & -0.1
\end{array}\right], B=\left[\begin{array}{c}
0 \\
0.1
\end{array}\right], K=-\left[\begin{array}{ll}
3.75 & 11.5
\end{array}\right] .
$$

The results summarized in Table 1 show the efficiency of the method. For comparison, the approaches based on a discontinuous Lyapunov functional [Liu and Fridman, 2012] and a Lyapunov-Krasovskii functional Fridman [2010] are also given. We can see that the proposed formulation gives better results.

\section{QUADRATIC STABILITY ANALYSIS OF APERIODIC UNCERTAIN LINEAR IMPULSIVE SYSTEMS}

Due to the complex dependence of the matrix expression $\mathcal{I}(P, A, J, T)$ on the inter-impulse periods and on the matrix $A$ (this expression is convex in $J$ ), it is unlikely to obtain tractable sufficient conditions for uncertain systems. The proposed functional-based approach however allows to perform this in a quite simple way. Due to the complexity of product patterns in the LMIs conditions, an intermediary result making the conditions of Theorems 2 and 3 affine/convex in the system matrices is first provided. Based on this result, conditions for robust stability analysis of impulsive systems are stated.

\subsection{Alternative stability conditions}

The following technical result is necessary to prove the main result of this section.

Lemma 4. For some given matrices $\mathcal{M}=\mathcal{M}^{T}, \mathcal{Y}=\mathcal{Y}^{T}$ and $\mathcal{Z}$ of appropriate dimensions, the following statements are equivalent:

(1) The matrix inequality $\mathcal{M}-\mathcal{Z}^{T} \mathcal{Y} \mathcal{Z} \prec 0$ holds.

(2) There exists a matrix $\mathcal{X}$ and a scalar $\mu>0$ such that the matrix inequality

$\mathcal{M}+\mathcal{X}^{T} \mathcal{Z}+\mathcal{Z}^{T} \mathcal{X}+\mathcal{X}^{T}(\mu I+\mathcal{Y})^{-1} \mathcal{X}+\mu \mathcal{Z}^{T} \mathcal{Z} \prec 0$ holds with $\mu I+\mathcal{Y} \succ 0$.

Proof : The proof is inspired from [Briat, 2011] and is detailed in Appendix A, for readability. 


\begin{tabular}{|l|l|l|l|l|}
\hline Periodic Case & Ex.1 & Ex.2 & Ex.3 & Ex.4 \\
\hline \hline Theoretical upper bound & $(0,0.4620]$ & {$[1.140, \infty)$} & {$[0.1824,0.5776]$} & $(0,1.7294]$ \\
[Briat and Seuret, 2012] & $(0,0.4471]$ & {$[1.232, \infty)$} & {$[0.1824,0.5760]$} & $(0,1.7239]$ \\
Theorem 2 & $(0,0.4519]$ & {$\left[1.174,10^{6}\right]$} & {$[0.1824,0.5764]$} & $(0,1.7293]$ \\
Theorem 1 [Liu and Fridman, 2012] & - & - & - & $(0,1.36]$ \\
\hline \hline Aperiodic Case & Ex.1 & Ex.2 & Ex.3 & Ex.4 \\
\hline [Briat and Seuret, 2012] & $0,0.4471]$ & {$[1.232, \infty)$} & {$[0.1907,0.5063]$} & $(0,1.7239]$ \\
Theorem 3 & $(0,0.4483]$ & {$\left[1.232,10^{6}\right]$} & {$[0.1824,0.5741]$} & {$[0,1.7288]$} \\
Theorem 1 [Liu and Fridman, 2012] & - & - & - & $(0,1.36]$ \\
Theorem 1 [Fridman, 2010] & - & - & - & $(0,1.69]$ \\
\hline
\end{tabular}

Table 1. Allowable dwell-times for the considered systems.

We then have the following alternative formulation for Theorem 3.

Theorem 5. The impulsive system (1) with $T_{k} \in\left[T_{\min }\right.$, $\left.T_{\max }\right], \varepsilon<T_{\min }<T_{\max }<\infty, \varepsilon>0$, is asymptotically stable if one of the following equivalent statements hold.

(1) The conditions of Theorem 3 are satisfied.

(2) There exist matrices $P, Z \in \mathbb{S}_{+}^{n}, P_{0}, S, Q \in \mathbb{S}^{n}$, $U \in \mathbb{S}^{2 n}, R \in \mathbb{R}^{n \times 2 n}, N, X \in \mathbb{R}^{n \times 3 n}$ and a scalar $\epsilon>0$ such that the LMIs

$$
\Upsilon(A, J, T) \prec 0, \Gamma(A, J, T) \prec 0
$$

hold with $Q_{\epsilon}=\epsilon I+Q-P-P_{0} \succ 0$ for all $T \in\left\{T_{\min }, T_{\max }\right\}$ and all $i=1, \ldots, N$ where

$$
\begin{aligned}
\Upsilon(A, J, T) & =\left[\begin{array}{ccc}
\Upsilon_{0}(A, J, T) & X^{T} & \epsilon\left(J M_{-}\right)^{T} \\
\star & -Q_{\epsilon} & 0 \\
\star & \star & -\epsilon I
\end{array}\right] \\
\Gamma(A, J, T) & =\left[\begin{array}{cccc}
\Gamma_{0}(A, J, T) & T N^{T} & X^{T} & \epsilon\left(J M_{-}\right)^{T} \\
\star & -T Z & 0 & 0 \\
\star & \star & -Q_{\epsilon} & 0 \\
\star & \star & \star & -\epsilon I
\end{array}\right]
\end{aligned}
$$

where

$$
\Upsilon_{0}(A, J, T)=G_{0}(A, J, T)+T\left(G_{2}(A, J)+F_{3}\right)
$$$$
\Gamma_{0}(A, J, T)=G_{0}(A, J, T)+T\left(G_{1}(A)-F_{3}\right)
$$

and

$$
\begin{aligned}
& G_{0}(A, J, T)=T \operatorname{He}\left\{M_{x}^{T}\left(P+P_{0}\right) A M_{x}\right\}-M_{x}^{T} Q M_{x} \\
& \quad-\operatorname{He}\left[\left(M_{x}-J M_{-}\right)^{T}(R G+N)\right]-M_{+}^{T} P_{0} M_{+} \\
& \quad-M_{-}^{T} P M_{-}+\operatorname{He}\left[\left(X+M_{x}^{T} Q\right)^{T} J M_{-}\right] \\
& \begin{aligned}
G_{1}(A) & =\operatorname{He}\left[M_{x}^{T} A^{T} X M_{\varsigma}\right] \\
G_{2}(A, J) & =\operatorname{He}\left[M_{x}^{T} A^{T} Q\left(M_{x}-J M_{-}\right)+M_{x}^{T} A^{T} R G\right] \\
& +M_{x}^{T} A^{T} Z A M_{x} .
\end{aligned}
\end{aligned}
$$

If one of the above statements hold, then the positive definite quadratic form $V(x)=x^{T} P x$ is a Lyapunov function for the uncertain system (1) and we have

$$
\mathcal{I}(P, A, J, \theta) \prec 0
$$

for all $\theta \in\left[T_{\min }, T_{\max }\right]$.

Proof: The main difficulty in the conditions of Theorem 2 arises from the nonlinear terms $-M_{\zeta}^{T} Q M_{\zeta}$ and $M_{-}^{T} J^{T}(P+$ $\left.P_{0}\right) J M_{-}$. By noting that $M_{\zeta}=M_{x}-J M_{-}$, we get that

$$
\begin{aligned}
-M_{\zeta}^{T} Q M_{\zeta}+ & M_{-}^{T} J^{T}\left(P+P_{0}\right) J M_{-}= \\
& -M_{x}^{T} Q M_{x}+\operatorname{He}\left[M_{x} Q J M_{-}\right] \\
& -M_{-}^{T} J^{T} \bar{Q} J M_{-}
\end{aligned}
$$

where $\bar{Q}:=Q-P-P_{0}$. Since the last quadratic term has a central matrix $\bar{Q}=Q-P-P_{0}$ that is symmetric but indefinite, it is not possible to use Schur complement. To overcome this problem, we invoke Lemma 4 which infers the existence of a matrix $X$ and a scalar $\epsilon \geq 0$ such that we have

$$
\begin{aligned}
-M_{-}^{T} J^{T} \bar{Q} J M_{-}= & \operatorname{He}\left[X^{T} J M_{-}\right]+X^{T}(\epsilon I+\bar{Q})^{-1} X \\
& +\epsilon\left(J M_{-}\right)^{T}\left(J M_{-}\right),
\end{aligned}
$$

with $\epsilon I+\bar{Q} \succ 0$. Substituting the previous equation into (11) and then into the LMIs (4), performing Schur complements yields the result. Since the manipulations are lossless, the equivalence of the results follows.

The main interest for considering the alternative conditions stated in Theorem 5 lies in the fact that they are linear with respect to $J$ and convex with respect to $A$. These properties can be utilized to derive results for uncertain systems.

\subsection{Ranged dwell-time for uncertain systems}

We assume here that the matrices of the system (1) belong to the convex polytopes:

$$
\begin{aligned}
& A \in \mathcal{A}:=\operatorname{co}\left\{A_{1}, \ldots, A_{N_{A}}\right\} \\
& J \in \mathcal{J}:=\operatorname{co}\left\{J_{1}, \ldots, J_{N_{J}}\right\}
\end{aligned}
$$

where the matrices $A_{i}, i=1, \ldots, N_{A}$ are $J_{j}, j=1, \ldots, N_{J}$ are the vertices of the polytopes.

We are now ready to state the main result on stability of uncertain systems.

Theorem 6. Asume there exist matrices $P, Z \in \mathbb{S}_{+}^{n}$, $Q, S, P_{0} \in \mathbb{S}^{n}, U \in \mathbb{S}^{2 n}, R \in \mathbb{R}^{n \times 2 n}$ and $N, X \in \mathbb{R}^{n \times 3 n}$ and a scalar $\varepsilon \geq 0$ such that the LMIs

$$
\Upsilon\left(A_{i}, J_{j}, T\right) \prec 0 \text { and } \Gamma\left(A_{i}, J_{j}, T\right) \prec 0,
$$

hold for all $T \in\left\{T_{\min }, T_{\max }\right\}, i=1, \ldots, N_{A}$ and all $j=1, \ldots, N_{J}$.

Then, for any impulse sequence $\left\{t_{k}\right\}_{k \in \mathbb{N}}$ satisfying $t_{k+1}-$ $t_{k} \in\left[T_{\min }, T_{\max }\right]$, the system (1)-(12) is asymptotically stable and the inequality

$$
\mathcal{I}(P, A, J, \theta) \prec 0
$$

holds for all $(A, J, \theta) \in \mathcal{A} \times \mathcal{J} \times\left[T_{\text {min }}, T_{\text {max }}\right]$.

Proof : The proof is directly obtained from the convexity of the conditions stated in Theorem 5 .

Example 5. Let us consider an uncertain version of the system treated in Example 1 where we have

$$
\mathcal{A}:=\operatorname{co}\left\{\left[\begin{array}{cc}
1 & 3 \\
-1 & 2
\end{array}\right],\left[\begin{array}{ll}
2 & 2 \\
0 & 6
\end{array}\right]\right\} .
$$

In the periodic case, Theorem 6 yields the maximal period $T_{\max }=0.1149$ while an eigenvalue analysis yields the value 0.11555 , very close to the computed one. In the aperiodic case, i.e. ranged dwell-time, we use Theorem 6 
with $T_{\min }=0$ and we find $T_{\max }=0.1148$, which is the same result as in [Briat and Seuret, 2012].

Example 6. We revisit Example 3 where

$$
\mathcal{J}:=\operatorname{co}\left\{\left[\begin{array}{cc}
1.3 & 0 \\
0 & 0.25
\end{array}\right],\left[\begin{array}{cc}
1.1 & 0 \\
0 & 0.5
\end{array}\right]\right\} \text {. }
$$

Using Theorem 6 with periodic impulses, we find $T_{\min }=$ 0.2625 and $T_{\max }=0.5747$. This improves previous results since the interval $[0.2710,0.5198]$ was determined in [Briat and Seuret, 2012]. In the aperiodic case, the range of admissible dwell-times [0.2627, 0.5732] is obtained using Theorem 6 . These values are very close to the ones obtained in the periodic case. This demonstrates the relevance of the approach.

\section{CONCLUSION}

An improved version of the looped functional-based approach recently proposed in [Briat and Seuret, 2012] has been provided. The main advantage of the proposed approach is the characterization of discrete-time stability using a continuous-time criterion. This allows to incorporate more information on the interaction between the two parts of the system and to extend the results to aperiodic impulses and uncertain systems. Several examples have illustrated the benefits of the approach.

\section{Appendix A. PROOF OF LEMMA 4}

Define first

$$
\mathcal{I}:=\inf _{\mathcal{X}}\left\{\operatorname{He}\left[\mathcal{X}^{T} \mathcal{Z}\right]+\mathcal{X}^{T}(\mu I+\mathcal{Y})^{-1} \mathcal{X}+\mu \mathcal{Z}^{T} \mathcal{Z}\right\} .
$$

where the infimum is considered in the partially ordered set of symmetric matrices with partial order ' $\preceq$ '. To see the equivalence, it is enough to show the equality $\mathcal{I}=$ $-\mathcal{Z}^{T} \mathcal{Y} \mathcal{Z}$. Completing the squares yields

$$
\mathcal{H}^{T}(\mu I+\mathcal{Y})^{-1} \mathcal{H}-\mathcal{Z}^{T} \mathcal{Y} \mathcal{Z}
$$

where $\mathcal{H}:=\mathcal{X}+(\mu I+\mathcal{Y}) \mathcal{Z}$. Since $\mu I+\mathcal{Y} \succ 0$, the above quadratic term in $\mathcal{H}$ is convex, positive semidefinite and lower bounded by 0 . The lower bound is attained with the minimizer $\mathcal{X}^{*}=-(\mu I-\mathcal{Y}) \mathcal{Z}$ and therefore we have $\mathcal{I}=-\mathcal{Z}^{T} \mathcal{Y} \mathcal{Z}$. This concludes the proof.

\section{REFERENCES}

D.D. Bainov and P.S. Simeonov. Systems with impulse effects: Stability, theory and applications. Academy Press, 1989.

C. Briat. Convergence and equivalence results for the Jensen's inequality - application to time-delay and sampled-data systems. IEEE Transactions on Automatic Control, 56(7):1660-1665, 2011.

C. Briat and A. Seuret. Stability criteria for asynchronous sampled-data systems - a fragmentation approach. In 18th IFAC World Congress, pages 1313-1318, Milano, Italy, 2011.

C. Briat and A. Seuret. A boundary-constrainedfunctional approach for robust stability analysis of linear impulsive systems. submitted to Systems \& Control Letters, 2012.

C. Briat and E.I. Verriest. A new delay-SIR model for pulse vaccination. Biomedical signal processing and control, 4(4):272-277, 2009.
C. Cai, A. R. Teel, and R. Goebel. Smooth Lyapunov functions for hybrid systems Part ii: (pre)asymptotically stable compact sets. IEEE Transactions on Automatic Control, 53(3):734-748, 2008.

G. E. Dullerud and S. Lall. Asynchronous hybrid systems with jumps analysis and synthesis methods. Systems \& Control Letters, 37(2):61-69, 1999.

E. Fridman. A refined input delay approach to sampleddata control. Automatica, 46(2):421-427, 2010.

J.C. Geromel and P. Colaneri. Stability and stabilization of continuous-time switched linear systems. SIAM Journal on Control and Optimization, 45(5):1915-1930, 2006.

R. Goebel, R. G. Sanfelice, and A. R. Teel. Hybrid dynamical systems. IEEE Control Systems Magazine, 29(2):28-93, 2009.

J. P. Hespanha, D. Liberzon, and A. R. Teel. Lyapunov conditions for input-to-state stability of impulsive systems. Automatica, 44(11):2735-2744, 2008.

D. Liberzon. Switching in Systems and Control. Birkäuser, 2003.

K. Liu and E. Fridman. Wirtinger's inequality and lyapunov-based sampled-data stabilization. Automatica, 48(1):102-108, 2012.

A. N. Michel, L. Hou, and D. Liu. Stability of dynamical systems - Continuous, discontinuous and discrete systems. Birkhäuser, 2008.

P. Naghshtabrizi, J. P. Hespanha, and A. R. Teel. Exponential stability of impulsive systems with application to uncertain sampled-data systems. Systems $\&$ Control Letters, 57:378-385, 2008.

A. Seuret. Stability analysis for sampled-data systems with a time-varying period. In 48th Conference on Decision and Control, pages 8130-8135, Shanghai, China, 2009.

A. Seuret. A novel stability analysis of linear systems under asynchronous samplings. Automatica, 48(1):177182, 2012.

N. Sivashankar and P. P. Khargonekar. Characterization of the $\mathcal{L}_{2}$-induced norm for linear systems with jumps with applications to sampled-data systems. SIAM Journal on Control and Optimization, 32(4):1128-1150, 1994.

L. Stone, B. Shulgin, and Z. Agur. Theoretical examination of the pulse vaccination policy in the SIR epidemic model. Mathematical and computer modelling, 31(4-5): 201-215, 2000.

W. Sun, K. M. Nagpal, and P. P. Khargonekar. $H_{\infty}$ control and filtering with sampled measurements. In American Control Conference, 1991, pages 1652-1657, 1991.

Y. Yamamoto. New approach to sampled-data control systems - a function space method. In Proceedings of the 29th Conference on Decision and Control, Honolulu, Hawai, 1990, 1990.

T. Yang. Impulsive control theory. Springer-Verlag, 2001. 